\title{
Information Preservation (IP) Method in Simulation of Internal Rarefied Gas Flows in MEMS
}

\author{
Ching Shen \\ Laboratory of High Temperature Gas Dynamics \\ Institute of Mechanics, Chinese Academy of Sciences \\ 15 Zhongguancun Road, Beijing 100080 China
}

\begin{abstract}
This paper reviews firstly methods for treating low speed rarefied gas flows: the linearised Boltzmann equation, the Lattice Boltzmann method (LBM), the Navier-Stokes equation plus slip boundary conditions and the DSMC method, and discusses the difficulties in simulating low speed transitional MEMS flows, especially the internal flows. In particular, the present version of the LBM is shown unfeasible for simulation of MEMS flow in transitional regime. The information preservation (IP) method overcomes the difficulty of the statistical simulation caused by the small information to noise ratio for low speed flows by preserving the average information of the enormous number of molecules a simulated molecule represents. A kind of validation of the method is given in this paper. The specificities of the internal flows in MEMS, i.e. the low speed and the large length to width ratio, result in the problem of elliptic nature of the necessity to regulate the inlet and outlet boundary conditions that influence each other. Through the example of the IP calculation of the microchannel (thousands $\mu \mathrm{m}$ long) flow it is shown that the adoption of the conservative scheme of the mass conservation equation and the super relaxation method resolves this problem successfully. With employment of the same measures the IP method solves the thin film air bearing problem in transitional regime for authentic hard disc write/read head length $(L=1000 \mu m)$ and provides pressure distribution in full agreement with the generalized Reynolds equation, while before this the DSMC check of the validity of the Reynolds equation was done only for short $(L=5 \mu \mathrm{m})$ drive head. The author suggests degenerate the Reynolds equation to solve the microchannel flow problem in transitional regime, thus provides a means with merit of strict kinetic theory for testing various methods intending to treat the internal MEMS flows.
\end{abstract}

\section{INTRODUCTION}

Manufacture and application of the micro-electromechanical system (MEMS) commenced at $80^{\text {th }}$ of the $20^{\text {th }}$ century and constituted the frontiers of the development of science and technology. The spatial sizes of the flows in them are of the order of microns, the time scales are of the order of microseconds. The gas flows in them generally fall into the slip flow regime (the mean free path $\lambda$ under ordinary pressure is $\sim 0.065 \mu \mathrm{m}$ ), and the flows in micro-channel, micro-pump, micro-nozzle, micro-valve may enter into the transitional regime. There are various methods developed in the transitional regime backed by the powerful electronic computers. Some of them, including the method of linearised Boltzmann equation, the Lattice Boltzmann method (LBM), the Navier-Stokes equation plus slip boundary condition method and the direct simulation Monte Carlo (DSMC) method, will be introduced and reviewed in the next section. In particular, as shown by the check made by the DSMC method, the Lattice Boltzmann method (LBM) is unfeasible to solve the transition regime problems.

DSMC method is an appropriate method to treat the problems in this regime, but when the flow speed is very small, the information to noise ratio is very small, and leads to difficulties in statistical simulation. The information preservation (IP) method is introduced to overcome these difficulties, which is embedded in the framework of DSMC method and allows the simulated molecules to carry the macroscopic information of the enormous number of molecules one simulated molecule represents, uses it to obtain the macroscopic characteristics, and in principle has found the way to overcome these difficulties. This method is introduced in the section INFORMATION PRESERVATION (IP) METHOD with validation of the basic assumptions.

The specific features of low flow speed and large length to width ratio of internal flows also cause the problem of mutual influence of the inlet and outlet boundary conditions and the need to regulate them. We'll illustrate the way of successful resolving this boundary condition regulation problem by using the conservative scheme of mass conservation equation and the super relaxation method on the example of IP SIMULATION OF THE MICROCHANNEL FLOWS. These are the measures one should undertake when treating internal low speed rarefied flows.

By using the same measures the IP method is applied to THE THIN FILM AIR BEARING PROBLEM in transitional regime for authentic write/read head length $(\mathrm{L}=1000 \mu \mathrm{m})$, excellent agreement of the pressure

Invited paper on the topic "Internal Flows and Vacuum Systems"

CP762, Rarefied Gas Dynamics: $24^{\text {th }}$ International Symposium, edited by M. Capitelli (C) 2005 American Institute of Physics 0-7354-0247-7/05/\$22.50 
distribution is obtained with the result of generalized Reynolds equation with the flow rate of the Poiseuille flow provided by the linearized Boltzmann equation, while the previous DSMC simulation was done for calculating this problem only for very short head length $(\mathrm{L}=5 \mu \mathrm{m})$.

The author suggests THE USE OF THE DENERATED REYNOLDS EQUATION IN SOLVING THE MICROCHANNEL FLOW PROBLEMS with the flow rate of the Poiseuille flow calculated by the linearized Boltzmann equation. The calculation examples show good agreement with the experimental data, the results of DSMC and IP methods.

Finally, some review and summary are given.

\section{SEVERAL METHODS FOR SOLVING THE LOW SPEED RAREFIED GAS FLOWS IN MEMS}

\section{Method of linearized Boltzmann equation}

The linearized Boltzmann equation (see review and literature in [1]) is suitable for solution of low speed problems in MEMS, and can serve as the criterion for testing other methods. At the same time the linearized Boltzmann equation can be used to solve the flow field with temperature variation. Some times the equation being linearized is not the Boltzmann equation but the BGK equation [2], in which case the solution is much simpler. But to make the solution of the BGK equation corresponding to the physical reality, some modification of the kind of parameter regulation is needed.

\section{Lattice Boltzmann method}

At the second half of 1980 years Frish et al. developed the lattice gas method [3]. This method was improved by introducing the lattice Bolotzmann method (LBM, see [4] and the literature cited there). Recently Nie, Doolen and Chen [5] simulated the flows in microchannels under large Knudsen numbers using the LBM method and obtained results of the pressure distribution etc. The microchannel flows under the same parameters are simulated by us [6,7] using both Nie et al.'s LBM method and DSMC method, to examine the feasibility of the LBM method in the transitional regime. The simulation results show that for small Knudsen number $(K n=0.0194)$ the LBM and DSMC methods agree fairly well. For $K n=0.194$, the velocity of the LBM and the DSMC (as well the IP) methods differ slightly, but the pressure distribution results have apparent difference. In the transitional regime, when $K n=0.388$, The DSMC simulation results do not verify the negative deviation of the pressure from the linear distribution predicted by the LBM method, and the results of the LBM and DSMC differ significantly in magnitudes (see Fig.1). This shows clearly that the LBM method is not able to simulate the MEMS flows in transitional regime (see $[6,7])$.

\section{The Navier-Stokes Equation with Slip Boundary Conditions}

The solution of the rarefied gas dynamics problems by using the Navier-Stokes equation with slip boundary conditions can make advantage of the mature and efficient methods of the Computational Fluid Dynamics (CFD). Karniadakis and Sherwin developed high order finite element (spectra element) method [8] to solve the compressible and incompressible Navier-Stokes equations with the first and higher order slip boundary condition, and by using the so called $\mu$ Flow code solved many interesting MEMS flow problems which were also reported in [9]. Kardiadakis and Beskok extended the method for use in micro flows with Kn as high as 0.5 . This is a kind of extrapolation beyond the reasonable application range. Here we cite two examples with one showing the necessity of the caution and the other showing the success of the extrapolation. The first example is the calculation of mass flow rates through short micro channels [10]. The flow rates obtained by the slip Navier-Stokes method and the DSMC method (see Fig. 2) differs significantly as $K n>0.1$, and the former can not yield the Knudsen minimum. The second example is the flow in the air bearing between the write/read head and the hard disc drive platter. The slip corrected Reynolds equation can provide result in fair agreement with the DSMC result for $\mathrm{Kn}$ as high as 4.2 (see [11]). The agreement of the generalized Reynolds equation based on the solution of the Boltzmann equation by Fukui and Kaneko [12] is in excellent agreement with the result of DSMC. This latter success must attribute to the employment of the Boltzmann equation in obtaining the flow rate of the Poiseulle flow in the Reynolds equation which is appropriate in the entire transitional regime.

\section{The direct simulation Monte Carlo (DSMC) method}

The direct simulation Monte Carlo (DSMC) method [13] is an appropriate method to treat rarefied gas flows in MEMS. The simulation results of DSMC can be used as criteria for other methods and it is able to treat problems abundant physical contents. But utilization of DSMC method in flows of MEMS encounters with the problems of the excessively high demands to the storage and computation time of the computer. And it is a very 


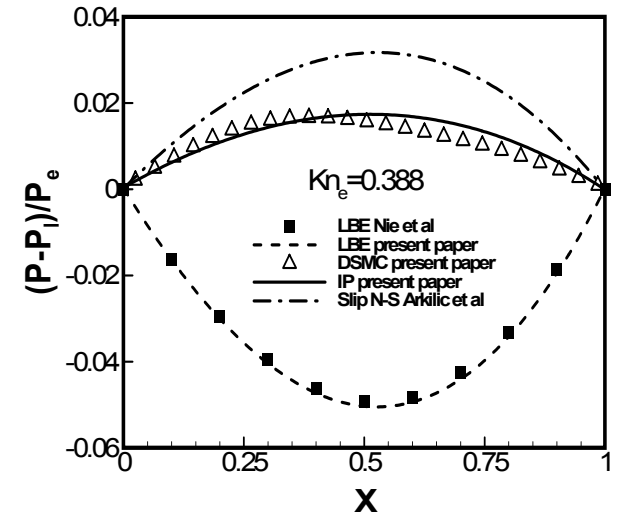

Fig.1 Deviation of pressure distribution from linearity Comparison of LBM, DSMC and IP simulation, $\mathrm{Kn}=0.388, p_{i} / p_{e}=2.0[6,7]$

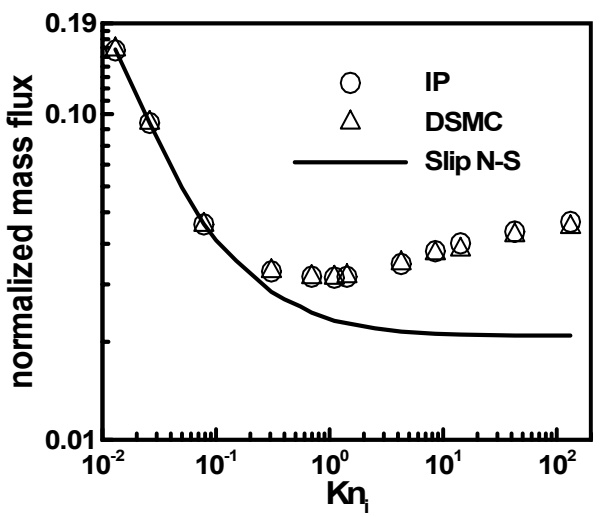

Fig. 2 The variation of the mass flux through short channels calculated by the IP, DSMC methods and the slip Navier-Stokes method [10].

Difficult task for DSMC to regulate the inlet and outlet boundary conditions of the long channel and other MEMS devises to gain the steadiness of the flow. This makes some researchers think that DSMC is not suitable for simulating gas flows inside MEMS [14]. In fact there have been many experimental results of the micro-channel flows [15-19], while the DSMC simulation of the micro channel flow has been limited to the high speed and even hypersonic cases [20,21].

\section{INFORMATION PRESERVATION (IP) METHOD}

Fan and Shen proposed a particle-based method, called the information preservation (IP) method [22, 23] to treat the difficulties encountered by the DSMC method. This is a method imbedded in the DSMC method in which each simulated molecule is assigned two velocities: thermal velocity $\boldsymbol{c}$ as in the DSMC method and information (IP) velocity $\boldsymbol{u}_{i}$ which is to record the collective velocity of the enormous number of real molecules represented by each simulated molecule. The IP velocities are used only for summation to obtain the macroscopic velocities. When the molecules reflect from the surface, collide with each other, experience force action and enter from boundary, the IP velocities attain new values (for more detailed information see [24, 25]):

1) For simulated molecules diffusely reflected, the reflected IP velocity $\boldsymbol{u}_{\boldsymbol{i}}$ has the velocity of the wall.

2) For two simulated molecules colliding with each other, the post-collision velocities attain the values

$$
u_{i, 1}^{*}=u_{i, 2}^{*}=\frac{m_{1} u_{i, 1}+m_{2} u_{i, 2}}{m_{1}+m_{2}}
$$

where superscript $*$ denotes post-collision quantities.

3) For simulated molecules entering the computational domain from boundaries, $\boldsymbol{u}_{\boldsymbol{i}}$ is set to satisfy the boundary condition.

4) In general under the isothermal assumption (which is valid for slow subsonic micro flows without heating) the IP velocity $\boldsymbol{u}_{i}$ of the simulated molecule and the IP velocity $\boldsymbol{U}$ and IP density $\rho$ (or n) of the cell are introduced which obey the mass conservation and momentum conservation equations

$$
\begin{gathered}
\iiint \frac{\partial \rho}{\partial t} d V=\iint \rho \vec{U} \cdot \vec{l} d S, \\
\iiint \rho \frac{d \vec{U}}{d t} d V=-\iint p \vec{l} d S,
\end{gathered}
$$

where the integrals are taken on the volume and surfaces of a cell, $\boldsymbol{l}$ is the external normal vector of the surface. It is noted that in the right hand side of the momentum equation only a non-viscous term is retained. In fact the IP quantities are governed by a general momentum equation

$$
\iiint \rho \frac{d \vec{U}}{d t} d V=-\iint \vec{\sigma} \vec{l} d S \equiv-\iint p \vec{l} d S+\iint \vec{\tau} \vec{l} d S
$$

where $\vec{\sigma}$ is the pressure stress tensor and $\vec{\tau}$ is the viscous stress tensor, for the IP quantities are carried along by the simulated molecules of the DSMC process which migrate across the cell surface in the positive and 
negative direction and implement the action of viscous transport. After a time step $\Delta t$ the cell IP density attains increment according to Eq. (2)

$$
\Delta \rho=-\frac{\Delta t}{\Delta V} \iint \rho \vec{U} \cdot \vec{l} d S
$$

from where the density and pressure are also renewed : $p=n k T$. The increment of the IP velocity of the cell is

$$
\Delta \vec{u}=-\frac{\Delta t}{\rho \Delta V} \iint \overrightarrow{p l} d S,
$$

and is added to the IP velocity of the simulated molecules in the cell. The renewed quantities are used for the next step calculation. The macroscopic velocity of a cell is obtained from the averaging of the IP velocities of molecules in the cell

$$
u_{0}=\frac{1}{N_{c}} \sum_{k=1}^{N_{c}} u_{i, k},
$$

where $N_{c}$ is the number of simulated molecules in the cell; $k$ is the index of the molecules in a cell.

\section{The validation of the method}

Now we validate the reflection rule 1) of the IP procedure [25]. As a simulated molecule represents an enormous number of real molecules, we trace the velocities of the numerous real reflected molecules and obtain the IP value by averaging. An individual molecule after diffuse reflection from a stationary surface would have velocity with the components $[13,24,25]$

$u=-(\ln (\operatorname{ranf}))^{1 / 2} / \beta, \quad v=V \cos \theta, \quad w=V \sin \theta$,

where $\beta=\left(2 \frac{k}{m} T_{w}\right)^{-1 / 2}, V=-(\ln (\operatorname{ranf}))^{1 / 2} / \beta, \theta=2 \pi \operatorname{ranf}$,

and ranf is a random fraction uniformly distributed between 0 and 1 . In the IP procedure we record the averaged values of $u, v$ and $w$. From the derivation of Eq. (8) and the practice of the DSMC procedure, one sees that thus sampled velocity components $u$ guarantee in the whole the correct value of the mass flux of diffusely reflected molecules and yield no macroscopic velocity in the normal to surface direction. So after averaging all $u$, the zero macroscopic velocity component is obtained:

$$
\bar{u}=0 \text {. }
$$

The averaging of $v$ (Eq. (8)) yields:

$$
\bar{v}=\overline{V \cos \theta}=\bar{V} \overline{\cos \theta}=0 \text {, }
$$

as $V$ and $\cos \theta$ are independent variates and $\overline{\cos \theta}=0$ according to Eq. (9). Similarly one has

$$
\bar{w}=0 \text {. }
$$

If the surface is not stationary but has certain velocity, the velocity components would have been added to $u, v, w$, and after averaging this velocity would be obtained as the IP velocity after diffuse reflection. So the statement "for simulated molecules diffusely reflected from the wall, the reflected IP velocity $\boldsymbol{u}_{i}$ has the same velocity as the wall" is validated.

Next we validate the IP collision rule $[25,26]$. The component $u_{1}^{*}$ of the post-collision velocity of one of the two collision partners (with velocity components $u_{1}, v_{1}, w_{1}$ and $u_{2}, v_{2}, w_{2}$ before collision) is found to be [25]

$$
u_{1}^{*}=\frac{m_{1} u_{1}+m_{2} u_{2}}{m_{1}+m_{2}}+\frac{m_{2}}{m_{1}+m_{2}} \sin \theta \cos \phi c_{r}^{*},
$$

where $c_{r}^{*}$ is the post-collision relative velocity, $\phi$ is a variate uniformly distributed between 0 and $2 \pi$, and $\cos \theta$ is a variate uniformly distributed between -1 and 1 . Here we understand $u_{1}, v_{1}, w_{1}, u_{2}, v_{2}, w_{2}$ as one set of the velocity components of many individual real molecules. We are intending to record (preserve) the averages of velocity components of the enormous number of molecules. We have from Eq. (13)

$$
\overline{u_{1}^{*}}=\frac{\overline{m_{1} u_{1}+m_{2} u_{2}}}{m_{1}+m_{2}}+\frac{m_{2}}{m_{1}+m_{2}} \overline{\sin \theta} \overline{\cos \phi} \overline{c_{r}^{*}}=\frac{m_{1} \overline{u_{1}}+m_{2} \overline{u_{2}}}{m_{1}+m_{2}},
$$

as the $\sin \theta$ and $\cos \phi$ are independent variates and $\phi$ is uniformly distributed between 0 and $2 \pi$ Thus, the IP collision rule, Eq. (1) of step 2), has been validated. 


\section{IP SIMULATION OF THE MICROCHANNEL FLOWS REGULATION OF THE INLET AND OUTLET BOUNDARY CONDITIONS}

Microchannel is the basic constituent of the MEMS devices, its geometric form is regular and simple, but it can reveal the specific features of the low speed micro internal flows, i. e., the issue of the mutual influence of the boundary conditions caused by the elliptic nature of the problem. For the DSMC-IP procedure it is necessary to prescribe the values of the pressure $p$ and the velocity distribution $\boldsymbol{U}$ over the cross sections at the inlet and the outlet of the channel to start any simulation. Arbitrarily chosen $p$ and $\mathbf{U}$ would contradict each other. The correct values of $p$ and $\boldsymbol{U}$ at inlet and outlet must be obtained in the process of solution of the problem. Here the pressure $p$ is fixed as the same of the prescribed (experimental) condition and $\boldsymbol{U}$ is allowed to change continuously and finally reach the steady solution. Thus the process of the DSMC-IP solution is always one of gradual adjustment towards a steady state (for more detailed elucidation of this problem see $[10,24,25]$. It is very critical that the conservative form of the mass conservation equation must be employed, with the second order central difference scheme yielding the density increment

$$
\Delta \rho=\Delta t\left(\frac{\rho_{i-1, j} U_{i-1, j}-\rho_{i+1, j} U_{i+1, j}}{2 \Delta x}+\frac{\rho_{i, j-1} V_{i, j-1}-\rho_{i, j+1} V_{i, j+1}}{2 \Delta y}\right) .
$$

This guarantees that the mass flux flown from the adjacent domain of a cell will flow without any numerical error into the integral area and vice versa and avoids the accumulation of numerical errors from the non-conservative scheme. The increments $\Delta \rho$ and $\Delta u$ from Eq. (15) and Eq. (6) allow one to obtain the renewed fields of $\rho u$ and $\rho v$ which are unfortunately with large fluctuations and are smoothened by an averaging technique to avoid the amplification of the errors. The increment $\Delta \rho$, Eq. (15), is only of the order of $10^{-9}$ of $\rho$ with $\Delta t$ being the order of the collision time for slow gas motion in long micro-channels [15-19]. Direct employment of this $\Delta \rho$ to achieve the steady (convergent) state is too time-consuming. A super-relaxation technique is employed to speed up the convergence process

$$
\rho_{i, j}^{t+\Delta t}=\rho_{i, j}^{t}+\omega \Delta \rho_{i, j}^{t},
$$

where $\omega$ is a relaxation factor. In practical calculations $\omega$ is taken to be between 100 and 2000 and trends to 1 when convergence is achieved. The effect of the acceleration action of the super-relaxation technique is illustrated on the experimental case [16] of nitrogen flowing in a $1.2 \times 30 \times 3000 \mu \mathrm{m}^{3}$ channel with inlet pressure of 15 psig into the atmosphere. Fig. 3 shows the evolution of the density $\rho$ at the center of the cross section located at $2500 \mu m$ from the inlet in the IP calculation by using $\mathrm{a}_{3}$ super-relaxation factor $\omega$ of 1,100 and 1000 , respectively. While $\rho$ approaches the steady value of $1.39 \mathrm{~kg} / \mathrm{m}$ in about $6 \times 10^{4}$ time steps with a relaxation factor $\omega$ of 1000 , the evolution for $\omega=100$ is further than halfway apart from the steady state after $6 \times 10^{4}$ time steps, and the value of $\rho$ remains almost the same when no super-relaxation is employed (with $\omega=1$ ). Fig. 4 shows the pressure distributions at inlet pressures of 8.7, 13.6 and 19.0 psig given by IP and experiment [17] in a $1.2 \times 40 \times 4000 \mu \mathrm{m}^{3}$ channel for helium with an exit Knudsen number of 0.16 , which agree well with each other. The IP simulation result will be verified by a more rigorous calculation based on the linearized Boltzmann equation in the penultimate section.

\section{THE THIN FILM AIR BEARING PROBLEM}

In a Winchester-type hard disc drive the write/read head floats approximately $50 \mathrm{~nm}$ above the spinning platter surface. The characteristic length (the height) is less than the mean free path ( $\sim 65 \mathrm{~nm})$ of molecules in air at STP. The typical Knudsen number is about 1.3. The platter speed is typically about $25 \mathrm{~m} / \mathrm{s}$ (at radius 5

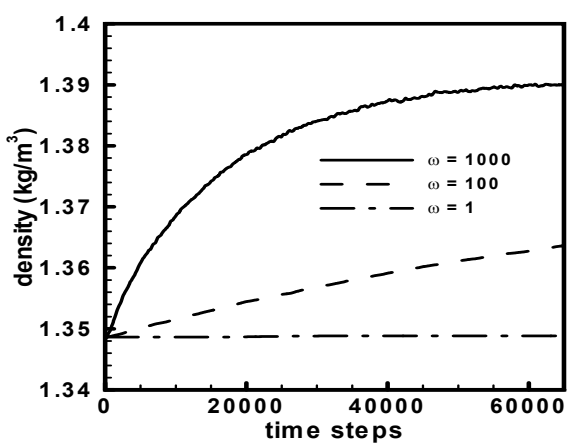

Fig. 3 The IP evolution processes of $\rho$ under conditions of [15] with $\omega=1, \omega=100, \omega=1000$, respectively. $\mathrm{cm}$ of the magnetic disc rotating at 4800 revolutions per minutes), corresponding to a Mach number of $\sim 0.07$ and a

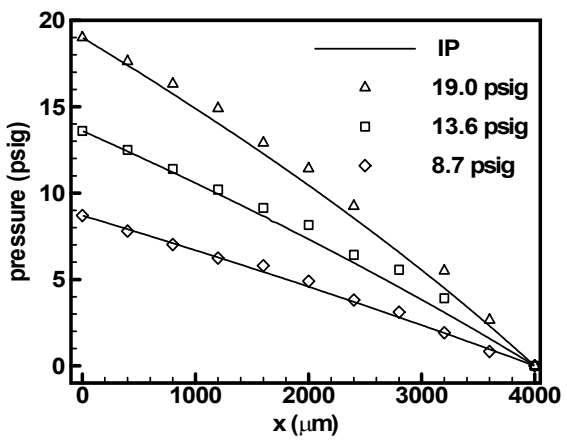

Fig. 4 Comparison of $\mathrm{p}$ distributions of helium flow given by IP and experimental data of [16]. 
Reynolds number of $\sim 0.12$. The typical length of the slider bearings in disc drives is $1 \mathrm{~mm}, \mathrm{i}$. e. , 20,000 times the gap at the rear edge of the head, and the width is usually $1 / 10-1 / 3$ of the length. The squeezed air bearing problem may be schematically modeled as a lower plate (the spinning platter) moving in its own plane with a velocity of $U$ under the upper stationary tilted plate (the write/read head, see Fig. 5). The thin film air flow between the plates is most appropriately described by the Reynolds equation which is a differential equation relating the pressure $p$, density $\rho$, platter velocity $U$ and the height $h$ of the gap, firstly developed by Reynolds for continuum fluid [27]. It is essentially a mass conservation relation applied not to a fluid element but to the cross sections of the squeezed air flow. This Equation for steady and two-dimensional case can be written in the normalized form [28]:

$$
\frac{d}{d X}\left(H^{3} P \frac{d P}{d X}\right)=\Lambda \frac{d}{d x}(P H)
$$

where $X=x / L, H=h / h_{o}, P=p / p_{o}$ and $\Lambda=6 \mu U L / p_{o} h_{o}{ }^{2}$ is the bearing number. In [25] it is shown that the equation (17) just reflects the fact that the flow rate across any cross section is the sum of the flow rate of the Couette flow (the right hand side) and the Poiseuille flow (the left hand side with the negative sign) and this rate does not change from one cross section to another in steady flow. Fukui and Kaneko [12] showed that the solution of the linearized Boltzmann equation for the thin film bearing problem can be decomposed into the solutions of the plane Couette and the Poiseuille flows and derived the generalized Reynolds equation which in the isothermal case can be written as

$$
\frac{d}{d X}\left[\bar{Q}_{P, T R}(K n) H^{3} P \frac{d P}{d X}\right]=\Lambda \frac{d}{d x}(P H),
$$

where $\bar{Q}_{P, T R}(K n)$ is the flow rate in transitional regime (normalized by the slip-less value $Q_{P, C}$ ) calculated from the linearized Boltzmann equation for Poiseuille flow and is shown to be the same as solved by Cernignani and Daneri [29]. A tabled database of the calculated values of $\bar{Q}_{P, T R}(K n)$ for $\sigma=0.7$ to $\sigma=1$ is provided in [30], and a fitted formula for $\sigma=1$ by Robert is recorded in [11]

$$
\bar{Q}_{P, T R}(K n)=1+6 A K n+\frac{12}{\pi} K n \log (1+B K n),
$$

where $A=1.318889$ and $B=0.387361$. Alexander, Garcia and Alder [11] used the DSMC method to simulate the short head length air bearing problem $\left(L=5 \mu \mathrm{m}, h_{o}=50 \mathrm{~nm}=0.05 \mu \mathrm{m}, U=25 \mathrm{~m} / \mathrm{s}, \sigma=1\right.$ and also under conditions of alternative combinations of the above parameters), and found excellent agreement of the DSMC simulation with the generalized Reynolds equation (18) (and Eq. (19) when $\sigma=1$ ). This just confirms that the generalized Reynolds can be used to solve the air bearing problem in the entire transitional flow regime. We note that the Winchester disc drive read/write head has an authentic length of $\sim$ $1000 \mu \mathrm{m}$, but the DSMC method for solving this problem was done only for short length $(\sim 5 \mu \mathrm{m})$.

The thin film air bearing problem is solved by the IP method in [31]. The rectangular area (from $x=0$ to $\mathrm{x}=\mathrm{L}$, and from $\mathrm{y}=0$ to $\mathrm{y}=\mathrm{h}(\mathrm{x}=0)$ ) is divided into rectangular uniform cells with some rectangular cells dissected by the upper plate. The incomplete cells are combined with their lower adjacent incomplete cells. In [31] a smoothing procedure is used to eliminate the no-physical oscillation. The weighted average value of the density itself in the cell and in the adjacent cells is used for smoothing. For example, for short heads ( $L=5 \sim 25 \mu \mathrm{m}$ ) limiting the weight of the cell itself between 0.996 and 0.9996 ensured the correct results without oscillation. The process of smoothing can be omitted if different time steps are used for the DSMC and the IP procedures: for DSMC the usual size of the time step of the order of collision time is sufficient, but for the IP simulation a smaller time step is used to ensure obtaining real macroscopic quantities of the solution. With the employment of appropriate super-relaxation factor steady convergent results can be obtained. Fig. 6 shows the comparison of the pressure distributions for the case of $L=1000 \mu \mathrm{m}$ of the IP result and the result of the generalized Reynolds equation with the use of the fitting formula Eq. (19). One can see that the agreement is excellent. The IP method is able to simulate the authentic length air bearing problem and can provide the detailed flow structure and with alternative boundary conditions (various $\sigma$ ).

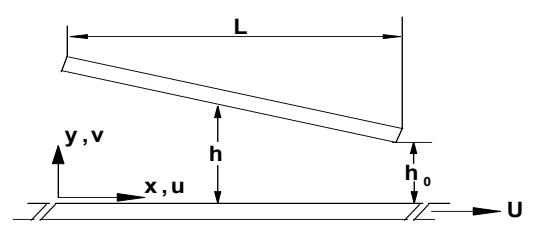

Fig. 5 A schematic model of the thin film air bearing flow

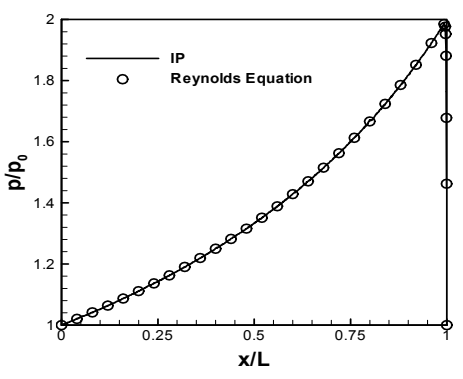

Fig. 6 Pressure distribution in the disc driver bearing for $K n_{o}=1.25, L=1000 \mu m$, comparison of IP and the generalized Reynolds equation results [31]. 


\section{USE OF THE DEGENERATED REYNOLDS EQUATION IN SOLVING THE MICROCHANNEL PROBLEM}

The generalized Reynolds equation (18) originally is derived for application in the thin film air bearing problem with the lower plate moving with a velocity $U$ and the upper plate tilted. We suggest use this Reynolds equation to solve the microchannel flow problem [25, 32]. Owing to the steadiness of the lower plate of the channel the right hand side term vanishes, as $U=0$ and $\Lambda=0$. Owing to the parallelity of the two plates the value $H$ is a constant and can be dropped from the equation. So the generalized Reynolds equation for application to the microchanel flow is degenerated to the form

$$
\frac{d}{d X}\left[\bar{Q}_{P, T R}(K n) P \frac{d P}{d X}\right]=0,
$$

where $\bar{Q}_{P, T R}(K n)$ is the flow rate for Poiseuille flow in transitional regime calculated from the linearized Boltzmann equation and normalized by the slip-less value $Q_{P, C}$. The values of $P$ on the inlet ad outlet of the channel are to be specified to make the microchanel problem solvable. For the case of diffuse reflectioin, the fitted formula approximation of $\bar{Q}_{P, T R}(K n)$, Eq. (19), can be used, and the degenerated Reynolds equation attains the form

$$
\frac{d}{d X}\left\{\left[1+6 A K n+\frac{12}{\pi} K n \log (1+B K n)\right] P \frac{d P}{d X}\right\}=0,
$$

The local Knudsen number $K n$ is most conveniently expressed through $P$, e. g., for hard sphere model

$$
K n=\frac{\lambda}{h}=\frac{C}{P},
$$

where

$$
C=\frac{\mu}{p_{o} h} \sqrt{\frac{\pi R T_{0}}{2}}=\lambda_{o} / h=K n_{o},
$$

$$
\text { for we have for hard sphere } \quad \lambda=\frac{\mu}{p} \sqrt{\frac{\pi R T_{o}}{2}},
$$

see, e.g., [25]. $\quad p_{0}$ is the pressure at the outlet, $T_{0}$ is the temperature of the gas, $\mu$ is the viscosity of the gas at $T_{0}$. The constant $C$ has the physical meaning of the Knudsen number at the outlet of the channel. Substituting Eq. (22) into Eq. (21), one arrives at

$$
\left[P+6 A C+\frac{12}{\pi} C \log \left(1+\frac{B C}{P}\right)\right] \frac{d P}{d X}=D,
$$

where $\mathrm{D}$ is an unspecified constant to be determined from the integration and has the physical meaning of the flow rate across the channel normalized by the slip-less flow rate value.

To illustrate the use of the degenerated Reynolds equation in solving the microchanel problem we calculate the pressure distribution for nitrogen in the $1.2 \times 40 \times 3000 \mu^{3}$ channel, and for helium in the $1.2 \times 40 \times 4000 \mathrm{\mu m}^{3}$ channel, compare the results with the experimental data of [16] and [17] and the IP calculation of [10]. For $T_{0}=294 K$ the value of $C=K n_{o}$ for helium is 0.15579 , and for nitrogen is 0.052325 . Eq. (25) is integrated under the following boundary conditions

$$
\left.P\right|_{X=0}=p_{\text {in }} / p_{\text {out }} \text {, and }\left.P\right|_{X=1}=p_{\text {out }} / p_{\text {out }}=1
$$

with $p_{i n}$ provided by the experimental data in $[16,17]$. The results of integration are presented in Fig. 7 and Fig. 8. From the comparison one witnesses the excellent agreement of the degenerated Reynolds equation, with the IP method (the curves almost coincide) and also the experimental data. This can be interpreted as the validation of the IP method by a calculation based on the linearized Boltzmann equation.

The degenerated Reynolds equation (21) with the flow rate $\mathrm{Q}_{\mathrm{P}, \mathrm{RR}}(\mathrm{Kn})$ provided by the linearized Boltzmann equation is appropriate for solving the microchanel flow problems and testing various methods in the entire transitional regime. From Eq. (25) it is seen that the microchannel rarefied gas flow is entirely determined by the specification of the inlet and outlet pressures $\mathrm{P}_{\text {in }}$ and $\mathrm{P}_{\text {out }}$, and the outlet Knudsen number $\mathrm{C}=\mathrm{Kn}_{\mathrm{o}}$, the length of the channel does not enter as a determining factor (remember, how much trouble the large length caused in the direct simulations). Database for the flow rates of the Poiseuille flow with various combinations of possible surface properties calculated on the basis of rigorous kinetic theory is desirable for the solution of the microchannel flow problem, the thin film air bearing problem and also the gas damping problem in micromechanical accelerometers [33], especially in the form of fitting formulas. 


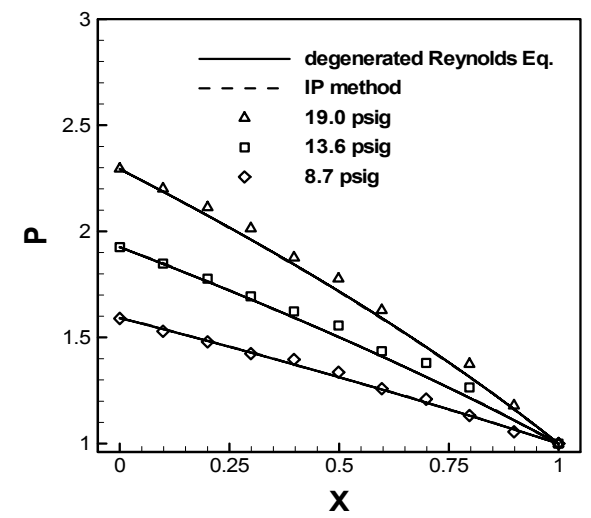

Fig. 7 Pressure distribution in microcannel flow of helium, comparison of the degenerated Reynolds equation, the IP and experimental Results [17].

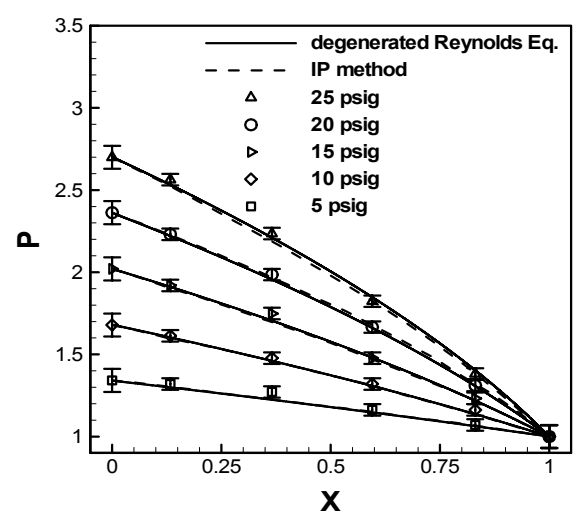

Fig. 8 Pressure distribution in microcannel flow of nitrogen, comparison of the degenerated Reynolds equation, the IP and experimental Results [16].

\section{REVIEW AND SUMMARY}

The information preservation (IP) method has been shown to be able to simulate the low speed rarefied gas flows in long (3000-7500 $\mu \mathrm{m})$ microchannels and hard disc drive bearing of authentic length $(1000 \mu \mathrm{m})$ with results in excellent agreement with the experimental data, the DSMC method and the generalized Reynolds equation and the degenerated Reynolds equation. As the latter are backed by the flow rates of Poiseuille flow calculated by the linearized Boltzmann equation, this can be considered as verification of the IP method in simulating 2-D micro flows by the strict kinetic theory. After this confirmation the IP method can be applied with certainty to MEMS flows of complicated configurations, which are easily treated by this method with the same flexibility as the DSMC method. The conservative scheme and the super-relaxation method are the necessary measures to be employed in solving all internal rarefied gas flows in MEMS.

The suggestion of using the degenerated Reynolds equation with flow rate of the Poiseulille flow calculated by the linearized Boltzmann equation to solve the microchannel flow provides a means with merits of strict kinetic theory for testing various methods intending to treat the internal MEMS flows. Database for the flow rates of the Poiseuille flow with various combinations of possible practical surface properties calculated on the basis of rigorous kinetic theory is desirable.

The IP method has been also used to simulate unidirectional and some 2-D and 3-D external flow problems with results in good agreement with theoretical investigation or experimental data which are not elucidated in this paper due to the limit of space. Interested readers may be referred to [24,25,34].

The extension of the IP method to the case of temperature variation is actual and interesting, there has been some attempts but with only partial success $[35,36,26]$. It remains a challenge to modify and develop the present IP algorithm to adapt it for the case of temperature variation.

Further theoretical validation of the basic assumptions of the method is also desirable.

\section{AKNOWLEDGEMENTS}

The support of the National Natural Science Foundation of China (Grant 90205024) is sincerely appreciated. The author expresses his gratitude for the invitation of the RGD Symposium Chairman Professor Mario Capetelli and the interest of the International Advisory Committee in his recent work and its indication to present an invited lecture on the topic "Internal Flows and Vacuum Systems".

\section{REFERENCES}

1. K. Aoki, AIAA Paper 2001-0874 (2001).

2. L.Bhatnagar, E.P.Gross,, M.A.Krook, Phys. Rev.,94,511-525(1954). 
3. U. Frish,B. Hasslacher, and Y. Pomeau, Phys. Rev. Let.,56,1505(1986)

4. S. Chen,S., G.D. Doolen,G.D., Annual Review of Fluid Mech.,30,329(1998).

5. X. Nie,G.D.Doolen,S. Chen, J. Statistical Physics 107,279(2002).

6. C.Shen,D.B.Tian,C.Xie,J.Fan, Proceedings of $1^{\text {st }}$ Intern conference on micro-mini-channels, ed. by S.G. Kandlikar, ASME, 405-410(2003). To appear in Microscale Thermophysics Engineering

7. Tian,D.B., Shen,C., Xie,C., Fan,J. Proceedings of the Seminar on frontier problems of the aerodynamics, 410-414 (2003).(in Chinese).

8. G.E. Karniadakis, S. Sherwin, Spectral/hp element method for CFD. Oxford Univ. Press, New York, (1999)

9. G. E. Karniadakis, A. Beskok, Micro flows, Fundamentals and simulation. Springer-Verlag, Heidelberg (2001)

10. C. Shen, J. Fan, C. Xie, J Comp Physics, 189,512-526 (2003)

11. F. J. Alexander, A. L. Garcia, B. J. Alder, Phys Fluids, 6:3854-3860 (1994)

12. S. Fukui, R. Kaneko, J Tribology, 110:253-262 (1988)

13. G. A. Bird, Molecular Gas Dynamics and Direct Simulation of Gas Flows, Clarendon press (1994)

14. E. S. Oran, C. K. Oh, B. Z. Cybek, Ann Rev Fluid Mech., 30:403 (1998)

15. J. Q. Liu, Y. C. Tai, K. C. Pong, C. M. Ho, The $7^{\text {th }}$ International Conference on Solid State Sensors and Actuators, Tranducers, 995-998 (1993)

16. K. C. Pong, C. M. Ho, J. Q. Liu, Y. C. Tai, ASME, FED 197, 51-56 (1994)

17. J. C. Shih, C. M. Ho, J. Q. Liu, Y. C. Tai, ASME-DSC 59, 197 (!996)

18. E. B. Arkilic, et al., in C Shen ed. Rarefied Gas Dynamics, Peking University Press, 983-988 (1997)

19. E. B. Arkilic, Measurement of the mass flow and the TMAC in silicon microchannels. Ph. D. Thesis, MIT, DFRL TR 97-1 (1997)

20. C. K. Oh, E. S. Oran, R.S. Sinkovits, J Thermophysics and Heat Transfer, 12,447 (1997).

21. R.P. Nance, D. Hash, H.A. Hassan, J Thermophysics and Heat Transfer, 11,497(1997)

22. J. Fan, C. Shen, C, Rarefied Gas Dynamics, edited by R Brun et al., Cepadues Editions, 2, 245-252 (1999)

23. J. Fan, C. Shen C, J Computational Physics, 167, 393-412 (2001)

24. C. Shen, Rarefied Gas Dynamics, National Defense Industry Press (2003), (in Chinese)

25. C. Shen, Rarefied Gas Dynamics, Springer Verlag (to appear)

26. J. Z. Jiang, C. Shen, J. Fan, Information preservation method with temperature variation, to appear (2004).

27. O. Reynolds, Philos Trans R Soc London, A177, 157-234 (1886)

28. W.A. Gross, L.A. Matsch, V. Castelli, A. Eshel, J.H. Vohr, M. Wildman, Fluid film lubrication. Wiley, New York (1980)

29. C. Cercignani, A. Daneri, J Applied Physics, 34:3509-3513 (1963)

30. S. Fukui, R. Kaneko, J Tribology, 112:78-83 (1990)

31. J. Z. Jiang, C. Shen, J. Fan, Statistical simulation of thin-film bearings. Paper presented at RGD-24, 10-16 July 2004, Bari, Itaty.

32. C. Shen, Use of the degenerated Reynolds equation in solving the microchannel flow problem, submitted to Physics of Fluids

33. T. Veijola, H. Kuisma, J. Lahdenpera, Sensors and Actuators, A48,239 (1995)

34. C. Shen, Proceedings of the 2003 seminar on frontier problems of the aerodynamics, China Serospace Press, 38-47 (2003). (in Chinese).

35. C. Shen, J.Z. Jiang, J. Fan, Rarefied Gas Dynamics, edited by T.J. Bartel and M.A. Gallis, AIP, 585: 185-192(2001)

36. Q. H. Sun, I.D. Boyd, J Computational Physics, 179:400-425 (2002) 